\title{
Editorial for September 2020 issue
}

\author{
S. V. Raghavan ${ }^{1,2}$
}

Published online: 16 July 2020

(c) CSI Publications 2020

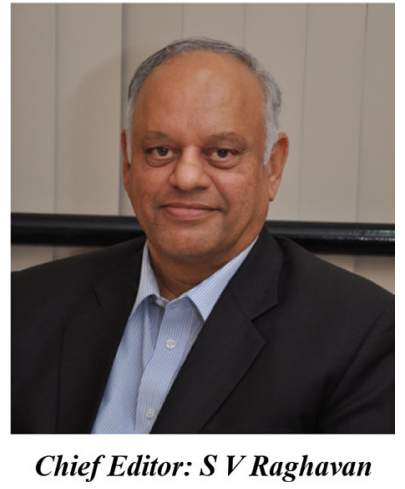

The September 2020 issue in your hands carries a series of interesting research work and simulations from young researchers across India. Some of the problems that require advancing the known solutions for efficiency and performance as well as finding novel methodologies for getting better accuracy and precision are, handwritten character and numerals recognition in various languages, ability to carry out secure multi-party communication ensuring privacy, efficient handling of IoT communication, and simple solutions to the integration of the complex world of medical imaging. We have articles in this issue that explore different aspects with open mind. I hope the readers find the approaches interesting and the results reported thought provoking. We are moving the journal into era of open

S. V. Raghavan

sv.raghavan@gov.in

1 CSI Transactions on ICT, Chennai 600036, India

2 Government of India, New Delhi, India exploration of complex information Technology problems. What you have is just the beginning and perhaps the tip of the iceberg. In my usual style, it is my pleasure to introduce briefly the six articles of this issue.

The paper titled, "Handwritten Kannada numerals recognition using deep learning convolution neural network (DCNN) classifier" by Hallur Vishweshwrayya and R $\mathrm{S}$ Hegadi explores the Kannada Numerals recognition to achieve an impressive classification process using deep convolution neural network classifier. The authors report an Isolation accuracy of $96 \%$ for Kannada numerals.

The paper titled, "An efficient multi-receiver certificate less digital multi-signature scheme with anonymity" by Tanwar Sarvesh and Anil Kumar propose an efficient Certificate-Less signature scheme called CL Digital MultiReceiver (CL-DMS) scheme that ensures anonymity without bilinear pairing, which is secure against forgeability, key replacement and key escrow attack. The authors also present an enhanced and efficient scheme, which is secure against Type 1, and Type II attacks and compare the proposed scheme with existing schemes in terms of computation and communication cost with higher level of security.

The paper titled, "A novel multi-objective optimizer framework for TDMA-based medium access control in IoT" by Pratap Siddavattam and Sedaghat Reza synthesizes and optimizes MAC layer of IoT based protocols using a novel delta diagram synthesizer and accurately captures both the high heterogeneity of the IoT and the impact of the Internet as part of the MAC layer architecture. This novel system saves considerable computation resources of IoT devices and rapidly adapts to various data sources with different distortion levels.

The paper titled, "Wireless sensor networks: a review of motes, wireless technologies, routing algorithms and static 
deployment strategies for agriculture applications" by Pawan Kumar and S R N Reddy presents a detailed literature review of various aspects of WSN design, starting from mote design and all the way to the associated application. The paper focuses on one of the most crucial application for livelihood i.e. crop production or agriculture domain.

The paper titled, "Costas Array Based Key Pre-Distribution Scheme (CKAPS) for WSN and its Performance Analysis" by Monjul Saikia and Md Anwar Hussain proposes a Costas Array based Key Pre-distribution Scheme (CAKPS). Comparative performance analysis of the scheme is shown for different grid sizes, while considering various order of Costas Array.
The paper titled, "A Distributed Architecture for Hospital Management Systems with Synchronized EHR" by Sumit Soman, Priyesh Ranjan, P K Srivatsava presents a Hospital Management Information Systems (HMIS) architecture which allows for integration of multiple standalone HMIS on a common platform that enables interoperability via Electronic Health Record (EHR) sharing and aggregation of standardized data for enabling analytics and visualization. Additionally, the proposed architecture also facilitates local, standalone deployments at primary medical facilities which are configured for local requirements and also synchronize essential datasets at the central node for enabling analytics.

Happy Reading... 